\title{
Actividad sinérgica in vitro de los alcaloides Crotsparina y Esparsiflorina aislados de Croton bomplandianum frente a Pseudomonas aeruginosa
}

\author{
Javier García, *Nelson Alvarenga \\ Universidad Nacional de Asunción, Facultad de Ciencias Química, Departamento de Fitoquímica, Dirección de \\ Investigación. San Lorenzo, Paraguay
}

Cómo referenciar este artículo/ How to reference this article:
García J, Alvarenga N. Actividad sinérgica in vitro de los alcaloides Crotsparina y Esparsiflorina aislados de Croton bomplandianum frente a Pseudomonas aeruginosa. Mem. Inst. Investig. Cienc. Salud. 2019; $17(1): 47-53$

\section{RE S U M E N}

Se evaluó la actividad sinérgica de los alcaloides crotsparina y esparsiflorina, aislados de Croton bomplandianum Baill. con los antibacterianos gentamicina y ciprofloxacina frente a Pseudomonas aeruginosa, microorganismo frecuentemente responsable de infecciones intrahospitalarias. Se empleó el método del "tablero de damas". Se encontraron combinaciones que presentaban efecto sinérgico, logrando la reducción de $87,5 \%$ de la CMI de gentamicina, mientras que para ciprofloxacina se logró una reducción del $25,0 \%$. Esto abre interesantes perspectivas sobre el uso combinado de productos naturales puros y fármacos en uso clínico para el tratamiento de infecciones producidas por este microrganismo.

Palabras clave: crotsparina, esparsiflorina, Croton, Pseudomonas, gentamicina, ciprofloxacina.

\section{In vitro synergic activity of the alkaloids Crotsparine and Sparsiflorine, isolated from Croton bomplandianum, against Pseudomonas aeruginosa}

\section{A B S T R A C T}

The synergistic activity of the alkaloids crotsparine and sparsiflorine, isolated from Croton bomplandianum Baill, was evaluated with the antibacterials gentamicin and ciprofloxacin against Pseudomonas aeruginosa, a microorganism frequently responsible for nosocomial infections. The checkerboard method was used. Combinations were found that had a synergic effect, achieving a reduction of $87.5 \%$ of the MIC of gentamicin, while for ciprofloxacin a reduction of $25.0 \%$ was observed. This opens interesting perspectives on the combined use of pure natural products and drugs in clinical use for the treatment of infections produced by this microorganism.

Keywords: crotsparine, sparsiflorine, Croton, Pseudomonas, gentamicin, ciprofloxacin.

\section{INTRODUCCIÓN}

Pseudomonas aeruginosa es uno de los más frecuentes microorganismos patógenos oportunistas responsable de infecciones intrahospitalarias ${ }^{(1)}$. Su importancia radica en que presenta en muchos casos resistencia múltiple a varios fármacos (MDR) así como su facilidad para adquirir resistencia en el curso del tratamiento, siendo inicialmente sensible a los antibacterianos utilizados ${ }^{(2)}$. El microorganismo se encuentra en los sistemas de agua hospitalarios, donde su capacidad de formar biofilms hace que su erradicación completa sea prácticamente imposible ${ }^{(3)}$. Está sindicado como responsable de neumonías intrahospitalarias y es frecuentemente aislado en pacientes con quemaduras infectadas ${ }^{(4)}$. Por lo antedicho, las infecciones por este microorganismo están asociadas a una alta tasa de

Fecha de recepción: diciembre 2018. Fecha de aceptación: febrero 2019

*Autor Correspondiente: Prof. Nelson L. Alvarenga Sosa. Departamento de Fitoquímica, Dirección de Investigación, Facultad de Ciencias Químicas de la Universidad Nacional de Asunción, Dr. José Decoud c/ Escuela Agrícola Mcal. López, Campus Universitario de San Lorenzo Km 9.5, San Lorenzo, Paraguay

Email: nelson@qui.una.py 
morbilidad/mortalidad y también a períodos prolongados de internación, con el consiguiente aumento de los costos de hospitalización ${ }^{(2)}$.

Por tanto, es importante la búsqueda de nuevas sustancias para tratar infecciones producidas por esta bacteria, solas o combinadas con los fármacos ya existentes, ya que una de las maneras de evitar o revertir la aparición de resistencia es la combinación sinérgica de dos o más agentes antibacterianos ${ }^{(5)}$.

Las plantas medicinales han sido utilizadas desde los albores de la humanidad para el tratamiento de diversas enfermedades y dolencias. Diversos compuestos han sido aislados, los cuales presentan variadas actividades biológicas, entre las cuales, la antibacteriana es una de ellas ${ }^{(6)}$. Entre los compuestos aislados, los alcaloides tienen una particular relevancia, debido al hecho de que normalmente presentan algún tipo de actividad biológica y que varios han demostrado poseer actividad antibacteriana ${ }^{(7)}$. En la literatura científica han empezado a aparecer artículos que tratan sobre el uso de productos naturales con antibacterianos en uso clínico como una de las alternativas para combatir el desarrollo de resistencia. Una ventaja añadida de esta aproximación es que permitiría poner en uso de nuevo fármacos que se han dejado de utilizar debido a la resistencia a ellos desarrollada y además el hecho de que las dosis necesarias en las combinaciones sinérgicas son mucho menores que las de los antibacterianos solos, lo cual reduciría los efectos adversos que varios de ellos poseen, algunos muy graves, como es el caso de los aminoglucósidos ${ }^{(8,9)}$.

El género Croton, perteneciente a la familia Euphorbiaceae, comprende alrededor de 1300 especies de árboles, arbustos y hierbas distribuidos en regiones tropicales y subtropicales de ambos hemisferios. La química del género es considerablemente diversa. Varias especies han sido identificadas como fuente de diferentes clases de alcaloides, un hecho que realza considerablemente la importancia del género desde el punto de vista medicinal ${ }^{(10)}$. Croton bomplandianum Baill es una planta nativa de América del Sur pero naturalizada en el subcontinente indio. De la misma, dos alcaloides mayoritarios han sido aislados, crotsparina y esparsiflorina ${ }^{(11)}$. La crotsparina es una proaporfina, mientras que la esparsiflorina es una aporfina (Figura 1).<smiles>COc1cc2c3c(c1O)C1(C=CC(=O)C=C1)C[C@H]3NCC2</smiles>

1<smiles>COc1cc2c3c(c1O)-c1cc(O)ccc1C[C@H]3NCC2</smiles>

2

Figura 1: Estructuras químicas de los alcaloides Crotsparina (1) y Esparsiflorina (2).

Ambos compuestos han demostrado poseer actividad antibacteriana de moderada a débil frente a varios microorganismos ${ }^{(12)}$.

Con estos antecedentes se ha decidido evaluar la actividad sinérgica de los alcaloides crotsparina y esparsiflorina con los antibacterianos gentamicina y ciprofloxacina. Ambos antimicrobianos 0 sus análogos se emplean en infecciones producidas por el microorganismo y la potenciación de sus efectos en caso de demostrarse sinergia, así como la disminución de las dosis puede retrasar la aparición de resistencia por parte de $P$. aeruginosa por un lado y minimizar los efectos adversos por el otro ${ }^{(13)}$. 


\section{MATERIALES Y MÉTODOS \\ Material vegetal}

Especímenes de $C$. bonplandianum fueron recolectados en Guarambaré, departamento Central, Paraguay, en abril de 2017. Las muestras fueron identificadas por investigadores del Departamento de Botánica de la FCQ y se depositó un ejemplar en el Herbario FCQ de la Facultad de Ciencias Químicas, Universidad Nacional de Asunción, Paraguay (R. Degen 3757).

\section{Reactivos}

Todos los reactivos fueron de grado analítico. Se emplearon los siguientes: ácido clorhídrico, diclorometano, metanol, etanol, hipoclorito de sodio, hidróxido de sodio y dimetil sulfóxido (Anedra, Buenos Aires, Argentina). La resazurina sódica fue de SigmaAldrich (St. Louis, MO, EUA), el caldo nutritivo Mueller-Hinton y el agar nutritivo MuellerHinton fueron de HiMedia Laboratories (Mumbai, India). La solución fisiológica se adquirió localmente. Para las cromatografías en capa fina (CCF) se emplearon el revelador óleum compuesto por una mezcla de ácido acético, agua y ácido sulfúrico en proporción 80:16:4, revelador Dragendorff compuesto por tetrayodobismutato de potasio y ácido acético (todos los reactivos fueron de Anedra, Argentina). Antimicrobianos: gentamicina y ciprofloxacina (Italquímica, Paraguay).

\section{Preparación del extracto}

El material vegetal se secó en una estufa con circulación de aire forzado, a $40{ }^{\circ} \mathrm{C}$, durante dos semanas. Las partes aéreas (hojas y ramas) fueron trituradas en un molino de cuchillas hasta llegar a un polvo fino. Este material $(500 \mathrm{~g})$ fue sometido a maceración durante 30 minutos con $\mathrm{HCl}$ al $5 \%$ en cantidad suficiente para cubrir completamente el polvo, con ayuda de un baño de ultrasonidos (ULTRASONS H-D, J.P. Selecta, España) a una temperatura de $25{ }^{\circ} \mathrm{C}$. Posteriormente el macerado fue filtrado a través de papel cuantitativo y el proceso se repitió dos veces más. Las tres porciones fueron reunidas y se agregó diclorometano en una proporción 3:1 en un embudo de separación para realizar la extracción. Este proceso se realizó tres veces. Las porciones orgánicas fueron separadas y desechadas. La porción acuosa se alcalinizó hasta pH 11 con hidróxido de sodio al $20 \%$ y se extrajo de nuevo con diclorometano, tres veces. Las porciones orgánicas fueron reunidas y el solvente fue eliminado en un evaporador rotatorio (RVO 400 SD Boeco, Alemania) a 40 ${ }^{\circ} \mathrm{C}$ para obtener el extracto crudo de alcaloides.

\section{Aislamiento de crotsparina y esparsiflorina}

El extracto de alcaloides se analizó inicialmente a través de una CCF con gel de sílice 60 como fase estacionaria (Merck KGaA, Darmstadt, Alemania), y una mezcla de diclorometano-metanol en proporción 9:1 como eluyente. La placa fue visualizada a la luz UV a dos longitudes de onda, 254 y $366 \mathrm{~nm}$, y posteriormente revelada con el reactivo Dragendorff para confirmar la presencia de los alcaloides. A fin de determinar la presencia de otros compuestos no alcaloides, el reactivo Óleum (un revelador universal) fue utilizado.

Para separar los componentes principales visualizados en la CCF, el extracto se sometió a cromatografía en columna de forma repetida, a presión atmosférica, con gel de sílice como fase estacionaria (Merck KGaA, Darmstadt, Alemania, diámetro de partícula 0,063-0,2 mm) y una mezcla de diclorometano-metanol 9:1 como eluyente. La identificación fue realizada por CCF por comparación de los valores de relación de frente (Rf) con muestras auténticas, las cuales fueron previamente aisladas en nuestro laboratorio y caracterizadas espectroscópicamente ${ }^{(12)}$.

\section{Ensayos microbiológicos \\ Microorganismo utilizado}

Se empleó la cepa de Pseudomonas aeruginosa CECT 108 (Colección Española de Cultivos Tipo). Los microorganismos se mantuvieron a una temperatura de $-20{ }^{\circ} \mathrm{C}$ en caldo Müeller-Hinton con un $15 \%$ de glicerol.

\section{Preparación del inoculo de trabajo}

Para la preparación del inóculo de trabajo, se tomó una pequeña porción de colonia de la bacteria en condiciones asépticas para evitar cualquier contaminación, y se transfirió a un tubo de ensayos con tapa a rosca que contenía $10 \mathrm{~mL}$ de caldo de cultivo Mueller-Hinton. Se incubaron durante 24 horas a una temperatura de $37 \pm 1^{\circ} \mathrm{C}$. Las suspensiones de bacterias 
obtenidas fueron diluidas con caldo Mueller-Hinton hasta una turbidez equivalente a 0,5 de la escala de McFarland, utilizando para ello un turbidímetro. Estas suspensiones bacterianas contenían aproximadamente $1 \times 10^{8} \mathrm{UFC} / \mathrm{mL}$. Posteriormente fueron diluidas en una proporción 1:20 con solución salina estéril para producir una concentración final de $5 \times 10^{6}$ UFC/mL.

\section{Determinación de la Concentración Mínima Inhibitoria.}

Para la determinación de la concentración mínima inhibitoria fue utilizado el método de microtitulación en placa con resazurina modificado(14). Se emplearon placas de microtitulación de 96 pocillos, bajo condiciones asépticas en un sistema de cabina de seguridad biológica (Forma Scientific, Inc., Marietta, OH, EUA).

Primeramente fue determinada la concentración mínima inhibitoria de las sustancias de control gentamicina y ciprofloxacina, y luego se realizaron los ensayos con los alcaloides crotsparina y esparsiflorina. Un volumen de $100 \mu \mathrm{L}$ de la solución de alcaloides fue añadido a cada pocillo, por triplicado, en la primera columna de la placa. Se añadieron $100 \mu \mathrm{L}$ de las sustancias de control (gentamicina y ciprofloxacina) en cada pocillo y por duplicado, en la misma columna. En cada uno de los pocillos restantes se añadieron $50 \mu \mathrm{L}$ de caldo de cultivo. Con una micropipeta semiautomática multicanal se tomaron $50 \mu \mathrm{L}$ de los pocillos de la primera columna y se realizaron diluciones seriadas, de modo que en cada pocillo quedaron $50 \mu \mathrm{L}$ de solución de alcaloides en concentraciones descendentes. Las últimas alícuotas fueron desechadas. Posteriormente, a todos los pocillos se les añadieron $40 \mu \mathrm{L}$ más de caldo $\mathrm{MH}$ y $10 \mu \mathrm{L}$ de suspensión bacteriana. Las dos últimas columnas de la placa fueron destinadas al control de crecimiento y al control de esterilidad, respectivamente. Al control de crecimiento se le añadieron $50 \mu \mathrm{L}$ de caldo y $50 \mu \mathrm{L}$ de suspensión bacteriana, mientras que al control de esterilidad se le añadieron $100 \mu \mathrm{L}$ de caldo de cultivo sin microorganismo. El volumen final en cada pocillo fue de $100 \mu \mathrm{L}$.

Las placas fueron incubadas durante 24 horas a $37 \pm 1^{\circ} \mathrm{C}$, y una vez concluido este periodo, fueron añadidos $10 \mu \mathrm{L}$ de resazurina en cada pocillo. Fueron incubadas nuevamente durante 18 horas para observar los resultados finales. Cada prueba fue realizada por triplicado.

\section{Determinación del efecto sinérgico: método del "tablero de damas".}

La determinación del efecto sinérgico entre los alcaloides y la gentamicina y ciprofloxacina se realizó por el método de tablero de damas ${ }^{(15)}$. Se utilizaron placas de microtitulación de 96 pocillos en una configuración $8 \times 8$ (ocho filas y ocho columnas).

En el eje de abscisas se realizaron diluciones seriadas de esparsiflorina (o crotsparina, dependiendo del caso), mientras que en el eje de ordenadas se realizaron las diluciones de los antibióticos, generándose así combinaciones en diferentes proporciones de las sustancias, a las que posteriormente se agregó la suspensión de bacterias a ensayar. La ultima y penúltima columnas fueron destinadas al control de esterilidad y al control de crecimiento, respectivamente. Las placas fueron incubadas durante 24 horas a $37 \pm 1{ }^{\circ} \mathrm{C}$, y una vez concluido este periodo, se añadieron $10 \mu \mathrm{L}$ del indicador resazurina en cada pocillo. Fueron incubadas nuevamente durante 18 horas para observar los resultados.

Las muestras, los antibióticos y la suspensión bacteriana fueron preparados de la misma manera que en la determinación de la CMI, excepto que la concentración inicial de los antibióticos y los alcaloides en la primera fila o columna de cada uno fue 2xCMI.

La naturaleza de la interacción establecida entre ambas sustancias se determinó mediante el cálculo del índice fraccional de la concentración inhibitoria (FICI, por sus siglas en inglés), a partir de la Concentración Inhibitoria Fraccionaria (FIC) de cada componente utilizando la siguiente fórmula:

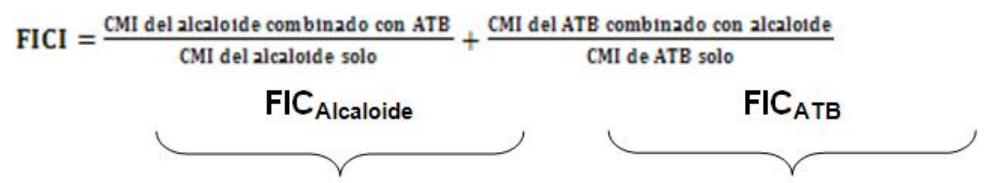

Los resultados se interpretaron de la siguiente manera:

- $\mathrm{FICI} \leq 0,5$ indica una interacción sinérgica entre las sustancias ensayadas.

- $0,5<\mathrm{FICI}<2$ indica una interacción aditiva.

- $\quad 2<\mathrm{FICI}<4$ indica que no existe interacción.

- FICI $>4$ indica una interacción antagonista. 


\section{RESULTADOS}

\section{Proceso de extracción}

La cantidad obtenida de extracto crudo de alcaloides fue de $1,5 \mathrm{~g}$, lo que representa un rendimiento de $0,02 \%$. La totalidad del extracto crudo de alcaloides fue sometido a cromatografía en columna para obtener los alcaloides puros. De este proceso se obtuvieron $40 \mathrm{mg}$ de crotsparina y $30 \mathrm{mg}$ de esparsiflorina.

\section{Determinación de la CMI}

Los resultados de la determinación de la CMI se muestran en la Tabla 1.

Tabla 1: Valores de CMI en $\mu \mathrm{g} / \mathrm{mL}$ para los antibacterianos y alcaloides frente a $P$. aeruginosa

\begin{tabular}{ll}
\hline Antibacterianos y alcaloides & Pseudomonas aeruginosa \\
Gentamicina & 1 \\
Ciprofloxacina & 0,5 \\
Crotsparina & 2000 \\
\hline Esparsiflorina & 2000 \\
\hline
\end{tabular}

\section{Ensayo de actividad sinérgica por el Método del Tablero de Damas}

Los resultados obtenidos con las combinaciones de los alcaloides y los antibióticos se presentan en la Tabla 2.

Tabla 2: Resultados obtenidos en el ensayo de sinergia por el método del tablero de damas, expresados en $\mu \mathrm{g} / \mathrm{mL}$ de la combinación entre gentamicina, ciprofloxacina, esparsiflorina y crotsparina frente a Pseudomonas aeruginosa.

\begin{tabular}{|c|c|c|c|c|c|}
\hline $\begin{array}{l}\text { Pseudomonas } \\
\text { aeruginosa }\end{array}$ & $\begin{array}{l}\text { CMI } \\
\text { individual }\end{array}$ & $\begin{array}{l}\text { CMI } \\
\text { combinada }\end{array}$ & FICI & $\%$ Red & Efecto \\
\hline $\begin{array}{l}\text { Gentamicina } \\
\text { Esparsiflorina }\end{array}$ & $\begin{array}{l}1 \\
2000\end{array}$ & $\begin{array}{l}0,125 \\
250\end{array}$ & 0,25 & $\begin{array}{l}87,5 \\
87,5\end{array}$ & Sinergismo \\
\hline $\begin{array}{l}\text { Ciprofloxacina } \\
\text { Esparsiflorina }\end{array}$ & $\begin{array}{l}0,5 \\
2000\end{array}$ & $\begin{array}{l}0,0625 \\
1000\end{array}$ & 0,625 & $\begin{array}{l}87,5 \\
50\end{array}$ & Aditivo \\
\hline $\begin{array}{l}\text { Gentamicina } \\
\text { Crotsparina }\end{array}$ & $\begin{array}{l}1 \\
2000\end{array}$ & $\begin{array}{l}0,125 \\
250\end{array}$ & 0,25 & $\begin{array}{l}87,5 \\
87,5\end{array}$ & Sinergismo \\
\hline $\begin{array}{l}\text { Ciprofloxacina } \\
\text { Crotsparina }\end{array}$ & $\begin{array}{l}0,5 \\
2000\end{array}$ & $\begin{array}{l}0,125 \\
250\end{array}$ & 0,375 & $\begin{array}{l}25 \\
87,5\end{array}$ & Sinergismo \\
\hline
\end{tabular}

\section{DISCUSIÓN}

Los resultados de los valores de CMI muestran que la cepa estudiada es sensible a ambos fármacos antibacterianos, aunque en el caso de gentamicina se encuentra en el límite, de acuerdo a los puntos de corte epidemiológicos (ECOFF) del Comité Europeo de Ensayos de Susceptibilidad Antimicrobiana (EUCAST) ${ }^{(16)}$.

Respecto a las pruebas de sinergia, los resultados obtenidos entre la combinación de gentamicina con esparsiflorina mostraron un marcado efecto sinérgico, con un valor de FICI de 0,25. La combinación consigue una reducción de la CMI del antibiótico y del alcaloide del $87,5 \%$. Esto es particularmente relevante en el caso de gentamicina, ya que es conocido que los antibióticos aminoglucósidos presentan importantes efectos adversos, siendo los más importantes la nefrotoxicidad y la ototoxicidad que muchas veces limitan su empleo ${ }^{(17,18)}$. Particularmente importante resulta el caso de la pérdida de audición debida al uso del fármaco, que puede llegar a ser irreversible. Respecto a la combinación de ciprofloxacina con esparsiflorina se observa efecto aditivo, no sinérgico. Sin embargo, a pesar de ello, se observa también una importante reducción en el valor de la CMI del 
antibacteriano que llega al $87,5 \%$. En donde el efecto es más modesto es en lo que respecta a la reducción de la CMI del alcaloide, que alcanza sólo el $50 \%$.

En relación a la combinación de los antibacterianos con la crotsparina, ambos mostraron efecto sinérgico. En el caso de la combinación con gentamicina, el valor de FICI obtenido fue de 0,25 y se observó una reducción en el valor de las CMI del antibiótico y el alcaloide de un $87,5 \%$. El resultado es similar al obtenido para la esparsiflorina, indicando que en el caso de la gentamicina la magnitud del efecto no es dependiente de las diferencias en la estructura del alcaloide.

Respecto a la combinación con ciprofloxacina, se observa un valor de FICI de 0,375 lo que evidencia un claro efecto sinérgico entre ambas sustancias. Se consigue una reducción del $25 \%$ en el valor de la CMI para la ciprofloxacina y un $87,5 \%$ en el de la crotsparina. La reducción obtenida en los valores de CMI de la ciprofloxacina con ambos alcaloides es relevante, ya que esta sustancia también presenta efectos adversos, si bien moderados en comparación con la gentamicina. No obstante, la reducción en el valor de la CMI implicaría eventualmente una reducción en las dosis empleadas del fármaco, aumentando su margen de seguridad ${ }^{(19)}$. A diferencia de los que sucede con la gentamicina, en el caso de la ciprofloxacina el efecto si depende de la estructura de los alcaloides, ya que en un caso se observa efecto aditivo y en el otro efecto sinérgico. Teniendo en cuenta que el antibacteriano es el mismo entonces la estructura del anillo D de los alcaloides, que es la porción diferente entre ambos, debe determinar de alguna manera que el efecto sea uno u otro. Esto requerirá de mayores estudios y la preparación de derivados a fin de establecer posibles relaciones estructura-actividad. Otra cuestión importante en relación a la interacción de la ciprofloxacina con los alcaloides es que la disminución de sus valores de CMI hace que se alejen del valor frontera de $0,5 \mu \mathrm{g} / \mathrm{mL}$ (por encima del cuál la cepa podría presentar resistencia), lo cual indica que los alcaloides aumentan la susceptibilidad del microorganismo al fármaco antibacteriano.

Los alcaloides crotsparina y esparsiflorina producen una importante disminución de los valores de CMI de los fármacos antibacterianos gentamicina y ciprofloxacina frente a $P$. aeruginosa. El efecto es sinérgico para la crotsparina con ambos fármacos y es sinérgico para la esparsiflorina con gentamicina y aditivo con ciprofloxacina. Estos resultados deben ser apoyados posteriormente por otros, como por ejemplo la realización de ensayos de curvas de letalidad o e-test. No obstante abren interesantes perspectivas sobre el empleo de fármacos en uso clínico con productos naturales puros para el tratamiento de infecciones producidas por $P$. aeruginosa y eventualmente otros microorganismos patógenos.

\section{AGRADECIMIENTOS}

A la Facultad de Ciencias Químicas de la Universidad Nacional de Asunción por brindar la infraestructura, materiales y condiciones para llevar adelante el trabajo. A la Prof. Rosa Degen del Departamento de Botánica de la FCQ por la identificación de los ejemplares de $C$. bomplandianum.

Fuentes de financiación: Fondos institucionales propios.

\section{REFERENCIAS BIBLIOGRAFICAS}

1. Feng W, Sun F, Wang Q, Xiong W, Qiu X, Dai $X$, et al. Epidemiology and resistance characteristics of Pseudomonas aeruginosa isolates from the respiratory department of a hospital in China. J Glob Antimicrob Resist. 2017;8:142-7.

2. Poole K. Pseudomonas aeruginosa: Resistance to the max. Front Microbiol. $2011 ; 2($ APR): $1-13$.

3. Wendel AF, Kolbe-Busch $S$, Ressina $S$, Schulze-Röbbecke R, Kindgen-Milles D, Lorenz $C$, et al. Detection and termination of an extended low-frequency hospital outbreak of GIM-1-producing Pseudomonas aeruginosa ST111 in Germany. Am J Infect Control. 2015;43(6):635-9.
4. Tissot F, Blanc DS, Basset P, Zanetti G, Berger MM, Que YA, et al. New genotyping method discovers sustained nosocomial Pseudomonas aeruginosa outbreak in an intensive care burn unit. J Hosp Infect. 2016;94(1):2-7.

5. Worthington RJ, Melander C. Combination Approaches to Combat Multi-Drug Resistant Bacteria. Trends Biotechnol. 2014;31(3):17784.

6. Mahady GB, Huang Y, Doyle BJ, Locklear T. Natural Products As Antibacterial Agents. Stud Nat Prod Chem. 2008;35:423-44.

7. Cushnie TPT, Cushnie B, Lamb AJ. Alkaloids: An overview of their antibacterial, antibiotic- 
enhancing and antivirulence activities. Int J Antimicrob Agents. 2014; 44(5):377-86.

8. Hemaiswarya $S$, Kruthiventi AK, Doble $M$. Synergism between natural products and antibiotics against infectious diseases. Phytomedicine. 2008;15(8):639-52.

9. Kuok C-F, Hoi S-O, Hoi C-F, Chan C-H, Fong $\mathrm{I}-\mathrm{H}, \mathrm{Ngok} \mathrm{C}-\mathrm{K}$, et al. Synergistic antibacterial effects of herbal extracts and antibiotics on methicillin-resistant Staphylococcus aureus : A computational and experimental study. Exp Biol Med. 2017;242(7):731-43.

10. Salatino A, Salatino MLF, Negri G. Traditional uses, chemistry and pharmacology of Croton species (Euphorbiaceae). J Braz Chem Soc. 2007;18(1):11-33.

11. Bhakuni DS, Satish S, Dhar MM. The alkaloids of croton sparsiflorus. Phytochemistry. 1970;9(12):2573-80.

12. Burgos A, Barua J, Flores-Giubi ME, Bazan $D$, Ferro $E$, Alvarenga NL. Antibacterial activity of the alkaloid extract and isolated compounds from Croton bonplandianum Baill. (Euphorbiaceae). Rev Bras Plantas Med. 2015;17(4).

13. Hurley MN, Cámara M, Smyth AR. Novel approaches to the treatment of Pseudomonas aeruginosa infections in cystic fibrosis. Eur Respir J. 2012;40(4):1014-23.
14. Sarker SD, Nahar L, Kumarasamy Y. Microtitre plate-based antibacterial assay incorporating resazurin as an indicator of cell growth, and its application in the in vitro antibacterial screening of phytochemicals. Methods. 2007;42:321-4.

15. Orhan G, Bayram A, Zer Y, Balci I. Synergy tests by $E$ test and checkerboard methods of antimicrobial combinations against Brucella melitensis. J Clin Microbiol. 2005;43(1):140-3. 16. European Committee on Antimicrobial Susceptibility Testing. Data from the EUCAST MIC distribution website [Internet]. 2018 [cited 2018 Sep 20]. Available from: https://mic.eucast.org/Eucast2/SearchControll er/search.jsp?action=performSearch\&BeginInd ex $=0 \&$ Micdif $=$ mic $\&$ NumberIndex $=50 \& A n t i b=-$ $1 \&$ Specium $=22$

17. Tulkens PM. Aminoglycosides: Nephrotoxicity. Antimicrob Agents Chemother. 1999;43(5):1003-12.

18. O'Sullivan $M E$, Perez $A$, Lin $R$, Sajjadi $A$, Ricci AJ, Cheng AG. Towards the Prevention of Aminoglycoside-Related Hearing Loss. Front Cell Neurosci. 2017;11(October):1-14.

19. Sarro A, Sarro G. Adverse Reactions to Fluoroquinolones. An Overview on Mechanistic Aspects. Curr Med Chem. 2001;8(4):371-84. 